\title{
Osteolytic and Osteoblastic Lesion of the Skull in a Patient with Known Cancer History: A Typical Location of Meningioma
}

\author{
Juan Antonio Alvarez Salgado*, Jorge Javier Villaseñor Ledezma, Maria de Los Angeles Cañizares \\ Menéndez, Luis Manuel Riveiro Vicente, Manuel Amosa Delgado, Francisco González-Llanos \\ Fernández de Mesa
}

Department of Neurosurgery, Virgen de la Salud Hospital, Toledo. Barber Av. 45001, Spain

Received: March 01, 2016; Accepted: April 13, 2016; Published: June 01, 2016

*Corresponding author: Juan antonio alvarez salgado. Department of Neurosurgery. Virgen de la Salud Hospital, Toledo. Barber Av. 45001, Spain, E-mail: alvsalju1@gmail.com

\section{Abstract}

Introduction: Meningiomas are the most common benign intracranial neoplasms. Although intraosseous meningiomas (IMs) are very rare, they are very well described in the literature. These lesions can be confused with metastatic carcinomas and bone tumors.

Case report: We present the case about man of 85 years old, underwent surgery with radical prostatectomy for prostate adenocarcinoma whose cranial tomography (CT) showed a parietotemporal tumor with extension to the left frontal bone formed by a combination of lytic and blastic areas, suggestive of metastatic lesion as first diagnosis. Cranial CT was requested extension study given the patient's underlying disease. The excision of the tumor and peritumoral bone fragment was necessary to reach an early diagnosis with subsequent cranial bone defect reconstruction using acrylic cement cranioplasty. The pathology is reported as intradiploic meningioma. The patient was discharged without presenting any neurosurgical complication.

Discussion: The IMs can be two types: primary or secondary. True primary type is defined as a lesion that does not involve the underlying dura. Secondary type is due to the extension of an intracranial meningioma into the calvarium. However, the underlying dura may be affected by primary or secondary IMs. Neurological signs and symptoms are usually absent in patients. The only curative treatment modality is complete resection. Cranial reconstruction must be done if the surgical resection is wide.

Conclusion: The osteolytic primary IM is rare but it should be considered in differential diagnosis of calvarial lesions even if the lesion seems hypermetabolic on PET scan and is diagnosed as metastasis. We recommend removing the single skull mass in patients with known cancer history.

\section{Introduction}

Meningiomas are the most common benign intracranial neoplasms. Although IMs are very rare, they are very well described in the literature. They are mostly seen in adults. Extradural meningiomas arise in locations other than the dura mater like neck, nasopharnyx or skin. Winkler, in 1904, first described a meningioma originating in an extradural location [1]. These lesions can be confused with metastatic carcinomas and bone tumors.

\section{Case Report}

We present the case about man of 85 years old, underwent surgery with radical prostatectomy for prostate adenocarcinoma, whose cranial CT showed a parieto-temporal tumor with extension to the left frontal bone formed by a combination of lytic and blastic areas, suggestive of metastatic lesion as first diagnosis. Cranial CT was requested extension study given the patient's underlying disease. The excision of the tumor and peritumoral bone fragment was necessary to reach an early diagnosis with subsequent cranial bone defect reconstruction using acrylic

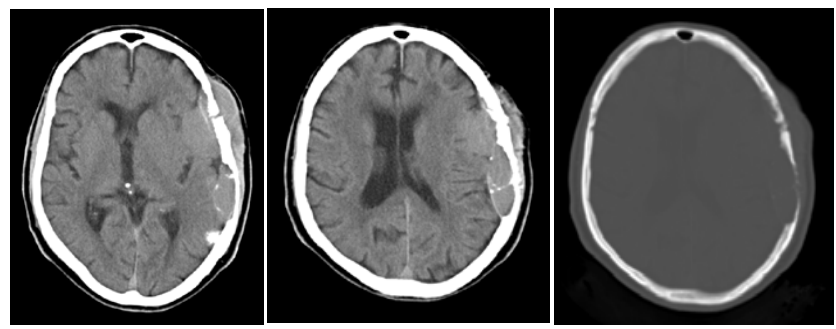

Figure 1: Preoperative cranial CT.

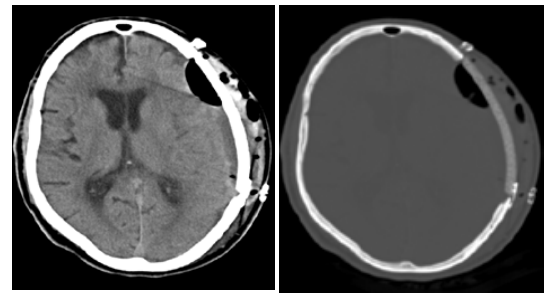

Figure 2: Postoperative cranial CT. 
cement cranioplasty. The pathology is reported as intradiploic meningioma. The patient was discharged without presenting any neurosurgical complication.

Classification scheme of primary extradural meningiomas*

\begin{tabular}{clc}
\hline \hline Type & \multicolumn{1}{c}{ Description } & Subclassification \\
\hline I & purely extracalvarial & not applicable \\
II & purely calvarial & B (skull base) \\
& & C (convexity) \\
III & calvarial with extracalvarial extension & B (skull base) \\
& & C (convexity) \\
\hline
\end{tabular}

* Adapted from Lang and colleagues, 2000.

\section{Discussion}

The etiology of IM has not been cleared yet. However, proposed theories include some presumed ectopic arachnoid cap cells in an extradural location, entrapment/detachment of displaced pacchionian bodies during embryonic development, displacement of arachnoid islets by a traumatic event or cerebral hypertension or a separate origin from a differentiated or multipotential mesenchymal cell.

The IMs can be two types: primary or secondary. True primary type is defined as a lesion that does not involve the underlying dura. Secondary type is due to the extension of an intracranial meningioma into the calvarium [2]. However, the underlying dura may be affected by primary or secondary IMs [3]

Lang and colleagues have proposed a classification for primary extradural meningiomas $\left({ }^{*}\right)$. In agreement with this clasisficacion the patient presented a tumor type IIIC. Frontoparietal and orbital regions are the most common locations for Ims. Neurological signs and symptoms are usually absent in patients. The initial symptom is usually a painless expansive mass with normal neurological findings. The symptoms are dependent on tumor location, size, and involvement of the neighboring structures.

Radiographic findings of IMs are limited and not pathognomonic [4]. The tumors are typically either osteoblastic or osteolytic, although mixed versions have been reported. Plain $\mathrm{X}$-ray skull radiographs can detect abnormalities, especially in the osteoblastic type. Hyperostosis, irregular foci of calcification, and atypical vascular marking can be seen. Normally these lesions do not show a 'dural tail'.

Histopathologically, these lesions may range from epithelial to mesenchymal in appearance. Although they are benign lesions, recent studies emphasize that intraosseous meningiomas have a higher incidence of malignant characteristics compared to intradural meningiomas $[5,6]$. The typical findings were found to be compatible with a meningothelial meningioma in our case.

The differential diagnosis of the osteoblastic type must include osteoma, osteosarcoma, Paget's disease, meningioma en plaque and fibrous dysplasia. The osteolytic subtype must be differentiated from hemangioma, chondroma, chondrosarcoma, dermoid, epidermoid tumor, brown tumor, multiple myeloma, plasmacytoma, giant cell tumor, aneurysmal bone cyst, eosinophilic granuloma, or metastatic cancer. The only curative treatment modality is complete resection. Cranial reconstruction must be done if the surgical resection is wide.

\section{Conclusion}

The osteolytic primary IM is rare but it should be considered in differential diagnosis of calvarial lesions even if the lesion seems hypermetabolic on PET scan and is diagnosed as metastasis. We recommend removing the single skull mass in patients with known cancer history.

\section{References}

1. Pompili A, Caroli F, Cattani F, Iachetti M. Intradiploic meningioma of the orbital roof. Neurosurgery. 1983;12(5):565-568.

2. Azar-Kia B, Sarwar M, Marc JA, Schechter MM. Intraosseous meningioma. Neuroradiology. 1974;6(5):246-253. DOI: 10.1007/ BF00345784.

3. Kudo H, Maeda S, Takamoto T, Tamaki N. Intraosseous meningioma with a dural defect. Neurol Med Chir (Tokyo). 1998;38(4):229-231.

4. Geoffray A, Lee YY, Jing BS, Wallace S. Extracranial meningiomas of the head and neck. AJNR Am J Neuroradiol. 1984;5:599-604.

5. Partington MD, Scheithauer BW, Piepgras DG. Carcinoembryonic antigen production associated with an osteolytic meningioma. Case report. J Neurosurg. 1995;82(3):489-492.

6. Younis G, Sawaya G. Intracranial osteolytic malignant meningiomas appearing as extracranial soft-tissue masses. Neurosurgery. 1992;30(6):932-935. 